\title{
Asthma diagnosis and treatment - 1004. The utility of mannitol challenge in the assessment of asthma
}

\author{
Dario Antolin-Amerigo*, Maria Jose Sanchez-Gonzalez, Mercedes Rodriguez-Rodriguez, Jose Barbarroja-Escudero, \\ Melchor Alvarez-Mon
}

From 2nd WAO International Scientific Conference (WISC 2012)

Hyderabad, India. 6-9 December 2012

\section{Background}

Mannitol challenge is becoming a supplementary and suitable method for the assessment of asthma, commonly used for the diagnosis of Exercise-Induced Asthma, due to its safety and high specificity, demanding less equipment and time requirement. Some studies show a closer relationship between AHR (Airway Hyperresponsiveness) to mannitol and markers of airway inflammation compared with AHR to methacholine in a selected group of asthmatic subjects. We aimed to describe its suitability in a group of patients who were referred to our outpatient clinic with asthma suspicion.

\section{Methods}

Forty-eight patients over 14 years old from our Allergy Department with asthma symptoms (dyspnoea, cough or wheezing) enrolled in the study. Mannitol was inhaled according to manufacturer instructions (Dr. Anderson 9Step protocol). The study included skin prick testing with a battery of common aeroallergens and respiratory function tests. All patients gave written informed consent.

\section{Results}

Forty-eight patients (27 female; 21 male) with a mean age of 30.61 years old were enrolled in the study. Forty patients were atopic (95.24\%), showing at least one positive skin prick test and/or aeroallergen specific IgE. Two patients did not show any positive skin prick test nor aeroallergen-specific IgE. Twenty-two patients (45.83\%) had a positive Mannitol Test with a mean PD15 of 155 mg. Mean Fractional exhaled nitric oxide (FeNO) of the former resulted $36.1 \mathrm{ppb}$. Six patients (27.27\%) exhibited a rapid response to Mannitol challenge and were classified as severe AHR. Five patients (22.72\%) showed a moderate response to Mannitol (Steps 5-6). Eleven patients $(50 \%)$ exhibited a mild response to Mannitol (Steps 7-9). All patients who manifested AHR after Mannitol Test presented a rapid recovery $(<10$ minutes) to baseline FEV1 after Salbutamol inhalation. The mean time required to complete the test was 18 minutes.

\section{Conclusions}

Mannitol might be considered as a convenient method for AHR diagnosis, due to its good safety and time-saving profile, specificity and relatively low equipment requirement.

Published: 23 April 2013

\section{doi:10.1186/1939-4551-6-S1-P4}

Cite this article as: Antolin-Amerigo et al.: Asthma diagnosis and treatment - 1004. The utility of mannitol challenge in the assessment of asthma. World Allergy Organization Journal 2013 6(Suppl 1):P4. 